\title{
A DIRECT METHOD OF USING THE HODOGRAPH PLANE IN FLUID DYNAMICS
}

\author{
by A. G. MACKIE \\ (Received 5th February 1958)
}

\section{The Hodograph Method in Incompressible Flow}

The application of the hodograph method in problems in fluid dynamics dates back to the time of Helmholtz and Kirchhoff. The underlying principle is simple. It is in effect to rewrite the governing differential equations with the roles of the original dependent and independent variables reversed. Such a procedure is not uncommon in problems depending upon ordinary differential equations. For example, if the velocity of a particle in rectilinear motion is prescribed as a function of distance from a fixed point, the problem of finding the relation between its position and the time $t$ can be solved by one quadrature if $t$ is regarded as the dependent variable. In the two-dimensional, irrotational motion of an incompressible fluid we have the velocity components given by

$$
\begin{aligned}
& u=\frac{\partial \phi}{\partial x}=\frac{\partial \psi}{\partial y} \\
& v=\frac{\partial \phi}{\partial y}=-\frac{\partial \psi}{\partial x}
\end{aligned}
$$

where $\phi, \psi$ are respectively the velocity potential and the stream function and both are harmonic functions of $x$ and $y$. It is a simple matter to show that, regarded as a function of $u$ and $v, \phi$ and $\psi$ still satisfy Laplace's equation. We shall work primarily with the stream function $\psi$ so that we have

$$
\frac{\partial^{2} \psi}{\partial u^{2}}+\frac{\partial^{2} \psi}{\partial v^{2}}=0
$$

or in polar co-ordinates $q, \theta$,

$$
\frac{\partial^{2} \psi}{\partial q^{2}}+\frac{1}{q} \frac{\partial \psi}{\partial q}+\frac{1}{q^{2}} \frac{\partial^{2} \psi}{\partial \theta^{2}}=0
$$

It is, of course, preferable to solve hydrodynamical problems directly in the physical plane, that is to say, in the plane of $x, y$ variables. However, some problems cannot be formulated initially in this plane-particularly those involving "free streamlines" on which the pressure and consequently the velocity remain constant. For one thing, the shape of these streamlines cannot be predetermined and the fact that the boundary condition on them is nonlinear is an additional mathematical complication. Thus problems involving jets and wakes are most easily solved in the hodograph plane where the boundary value problem can be formulated and where the boundary conditions are linear. The usual method of solution is to map the $\phi, \psi$ plane on the $u, v$ plane by a series of transformations in which that of Schwarz and Cristoffel plays a prominent part.

E.M.S.-G 2 
This method, while it is mathematically very elegant, has a number of unsatisfactory features. From the point of view of instruction to students it is difficult to teach concisely with satisfactory rigour. In addition, manipulation with conformal mapping tends to make the student lose sight of the fundamental idea which is the solution of a real boundary value problem in the hodograph plane. However, apart from these purely technical objections, there is another more serious one. The use of the Schwarz-Cristoffel transformation and the powerful complex variable theory associated with it is limited in application to Laplace's equation. In recent years hodograph methods have been extensively used in problems arising in the study of motion of compressible fluids. The governing equation for $\psi$ is not now Laplace's

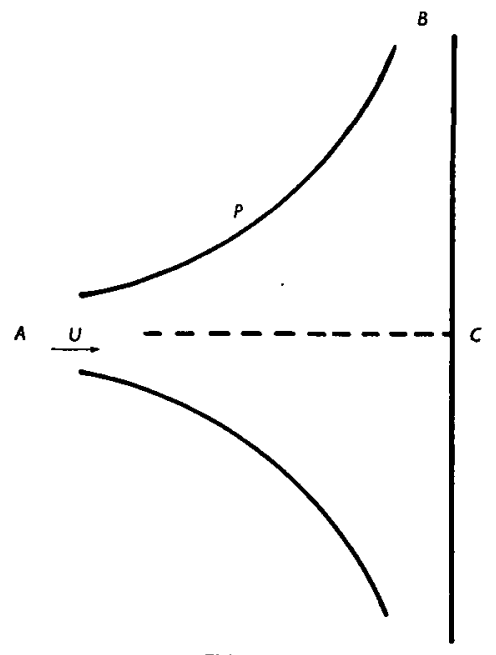

Fig. 1

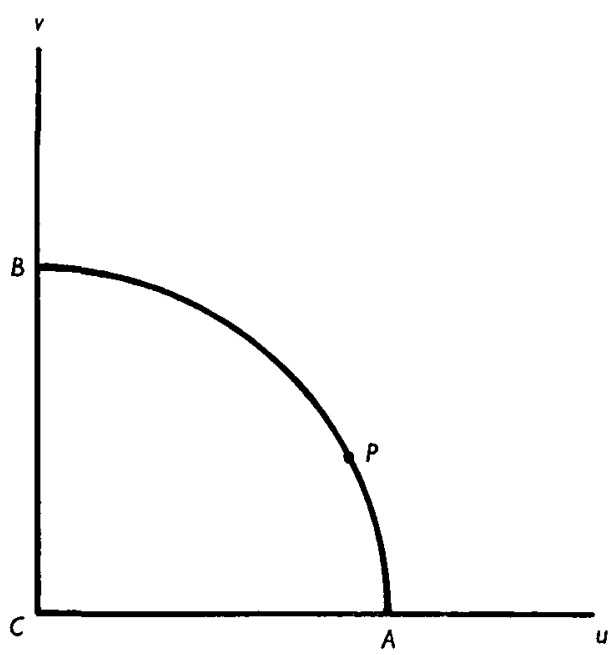

Fig. 2

equation and consequently the principle of Schwarz and Cristoffel can no longer be used. Workers in this field are therefore forced to work directly with real variables in the hodograph plane and to develop independent techniques. It seems highly desirable to apply these in the first place to the problems of incompressible flow as the mathematics is in general quite straightforward. We shall therefore describe by a number of examples how the basic problems for incompressible flow can be solved directly in the hodograph plane. As well as making the analysis simpler in several cases than that used in the method of Schwarz and Cristoffel this method serves as an easy and natural introduction to the use of hodograph methods in the treatment of compressible flow-both for the full hodograph equation and for the equation of Tricomi which approximates this equation in the neighbourhood of the speed of sound.

We begin with a simple example, that of the impact of a jet normal to a plane wall. We shall assume that the axis of the jet is at right angles to the wall although the case of oblique impact can be solved by a very minor extension. 
The physical and hodograph planes are shown in figs. 1 and 2 . It is sufficient to consider the upper half-plane only because of symmetry. The boundary conditions in the hodograph plane are $\psi=0$ on $C A$ and $C B$ and $\psi=K$ on APB which is part of the curve $q=U$. The value of $K$ is given by $K=h U$ where $h$ is the semi-width and $U$ is the velocity of the jet at infinity. It is clear that the solution of this Dirichlet problem is of the form

$$
\psi=K \sum_{n=1}^{\infty} a_{n}\left(\frac{q}{U}\right)^{2 n} \sin 2 n \theta
$$

which is a series of harmonic functions satisfying the boundary conditions on $C A$ and $C B$. In order to satisfy the boundary conditions on $A B$ we must have

$$
\sum_{n=1}^{\infty} a_{n} \sin 2 n \theta=1 \quad\left(0<\theta<\frac{1}{2} \pi\right)
$$

This gives

$$
\begin{aligned}
a_{n} & =\frac{4}{\pi} \int_{0}^{\frac{1}{2} \pi} \sin 2 n \theta d \theta \\
& =\frac{2}{n \pi}\left\{1-(-1)^{n}\right\} .
\end{aligned}
$$

Thus

$$
\psi=\frac{4 K}{\pi} \sum_{k=0}^{\infty} \frac{1}{2 k+1}\left(\frac{q}{U}\right)^{4 k+2} \sin (4 k+2) \theta .
$$

As a variation of this problem we can consider that of a jet impinging normally on a plate of finite width and breaking up into two jets inclined at an angle $2 \alpha$ to one another far downstream. The value of $\alpha$ will depend on the ratio of the jet width at infinity to the width of the plate. In this case the boundary conditions in the hodograph plane are $\psi=0$ on $C A, C B$ as before, $\psi=0$ on $B P$ and $\psi=K$ on $P A, \quad P$ is the point $(U, \alpha)$ in polar co-ordinates in the hodograph plane and maps into the point at infinity downstream from the plate in the physical plane. We seek a sine series as before but now we have to satisfy a step function boundary value on $q=U$. We obtain

$$
\psi=\frac{2 K}{\pi} \sum_{n=1}^{\infty} \frac{1}{n}(1-\cos 2 n \alpha)\left(\frac{q}{U}\right)^{2 n} \sin 2 n \theta .
$$

Suppose now we let $K \rightarrow \infty$ and $\alpha \rightarrow 0$ in such a way that $K \alpha^{2}$ remains finite. Then the width of the jet becomes infinite and we have the solution of the classical Helmholtz-Kirchhoff flow past a flat plate with a free stream breakaway from the trailing edges. This is

$$
\psi=c^{2} \sum_{n=1}^{\infty} n\left(\frac{q}{U}\right)^{2 n} \sin 2 n \theta .
$$

The value of $c^{2}$ is of no real significance as it depends on the width of the plate which is the only length parameter which appears. 
Other problems closely related to these are obtained when we consider not a flat plate but one consisting of two arms inclined to each other at an angle $\mu \pi$. The resulting wedge-shaped profile is then placed symmetrically with respect to the jet or main stream. In the complex variable approach it is usually pointed out that this can be reduced to the problem already considered by means of the transformation $\zeta=Z^{\mu}$. In our present approach we prefer to say that the problem is now one of fitting a Fourier sine series in the region $0<\theta<\frac{1}{2} \mu \pi$ instead of the range $0<\theta<\frac{1}{2} \pi$ as this is precisely the difference between the two cases in compressible flow also. In addition nonsymmetric problems can be easily solved. Of these the most important is

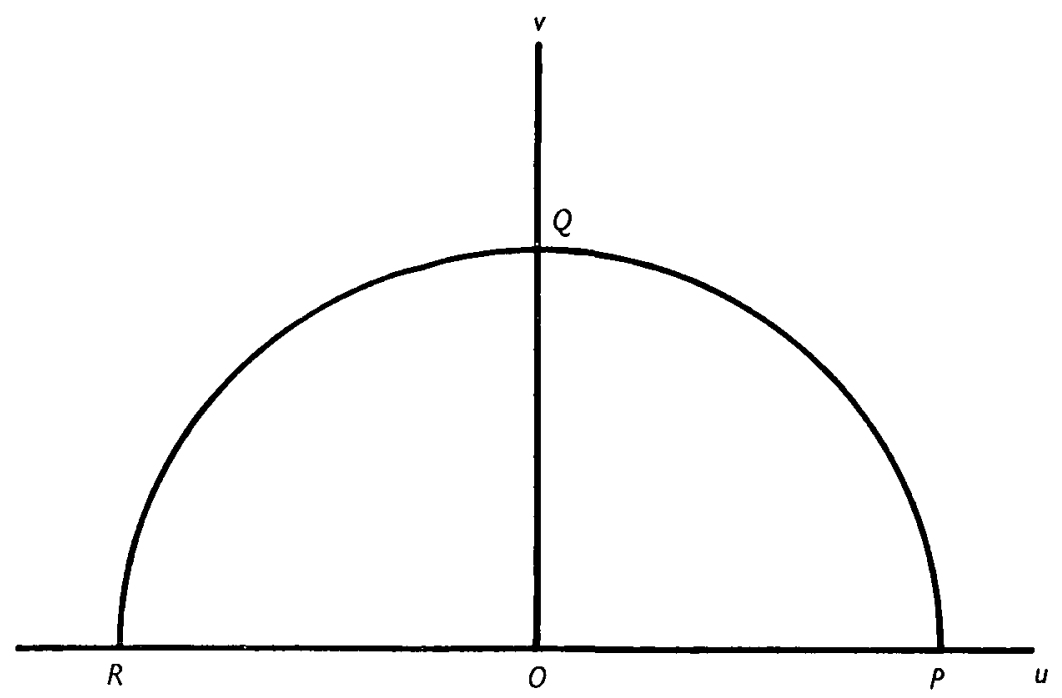

Fig. 3

probably that of flow against a plate which is inclined at an angle to the main stream where there is a free stream breakaway from the ends.

Another problem in which the boundary conditions can be readily set up in the hodograph plane is that of the Borda mouthpiece. Here there is a reservoir of fluid which is at rest at a sufficiently large distance from an exit consisting of a semi-infinite pipe with parallel sides which projects into the fluid. It is not difficult to see that one half of this flow maps into the upper semi-circle of the hodograph plane $P Q R O$ (fig. 3) and that the boundary conditions are $\psi=0$ on $O P, \psi=K$ on $O R$ and $P Q R$. This Dirichlet problem may be solved by using the Green's function for a semi-circle but it is easier to use the separation of variables solution as before, combined with the special solution $\psi=\theta$. Thus

$$
\psi=\frac{K \theta}{\pi}+K \sum_{n=1}^{\infty} a_{n}\left(\frac{q}{U}\right)^{n} \sin n \theta
$$




\section{THE HODOGRAPH PLANE IN FLUID DYNAMICS}

where

$$
\sum_{n=1}^{\infty} a_{n} \sin n \theta=1-\frac{\theta}{\pi} \quad(0<\theta<\pi)
$$

This gives

$$
\psi=\frac{K}{\pi}\left\{\theta+2 \underset{n=1}{\infty} \frac{1}{n}\left(\frac{q}{U}\right)^{n} \sin n \theta\right\} .
$$

We can easily modify this solution for the case of a pipe in which the sides are inclined at a general angle $\mu \pi$ to one another. When $\mu=1$ we have the problem of a jet issuing from a slit in a plane wall. Moreover it is possible to deal with a whole variety of different cases in which the exit is not symmetrical in relation to the pipe and these require the evaluation of the Fourier sine series of only the simplest functions.

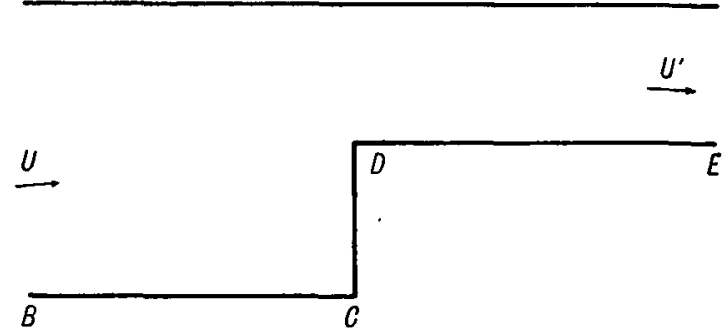

Fig. 4

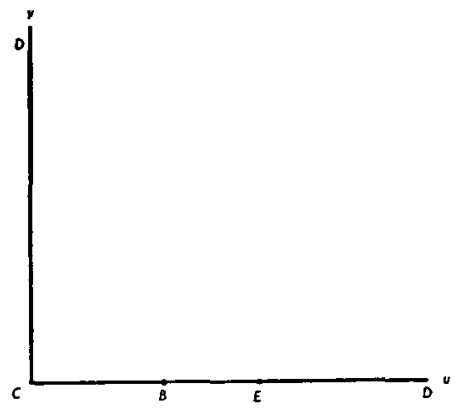

Fig. 5

For problems in which a body is placed in an infinite stream of undisturbed velocity $U$, the point $(U, 0)$ in the hodograph plane is a singular point as all streamlines must pass through it. The simplest example which illustrates the method of solution is that of flow past a semi-infinite rectangle whose side of finite length is at right angles to the stream. It is better to consider first the case in which the stream is contained in a channel of finite width.

The upper half of the physical plane and its mapping in the hodograph plane are shown in figs. 4 and 5. $\psi=0$ on $C B, E D$ and $C D$ and $\psi=K$ on $B E$. ( $B$ is the point $(U, 0)$ and $E$ the point $\left(U^{\prime}, 0\right)$ where $U^{\prime}$ is the velocity far downstream. $D$ is the point at infinity.) One way to solve this boundary value problem in the hodograph plane is by means of a Fourier sine transform. Thus

$$
\psi=\int_{0}^{\infty} f(\xi) \sin \xi u e^{-\xi v} d \xi
$$

where

$$
\int_{0}^{\infty} f(\xi) \sin \xi u d \xi=F(u)
$$


Here $F(u)=0$ for $0<u<U$ and $u>U^{\prime}$, and $F(u)=K$ for $U<u<U^{\prime}$. Hence

$$
\begin{aligned}
f(\xi) & =\frac{2 K}{\pi} \int_{U}^{U^{\prime}} \sin \xi u d u \\
& =\frac{2 K}{\xi \pi}\left(\cos \xi U-\cos \xi U^{\prime}\right) .
\end{aligned}
$$

Alternatively $\psi$ may be written immediately as

$$
\psi=\frac{K}{2 \pi} \int_{U}^{U^{\prime}}\left(\frac{\partial G}{\partial v^{\prime}}\right)_{v^{\prime}=0} d u^{\prime}
$$

where $G\left(u^{\prime}, v^{\prime} ; u, v\right)$ is the Green's function for the quarter-plane $u \geqslant 0, v \geqslant 0$. In this form we see the expression for the limiting solution without difficulty. As $K \rightarrow \infty$ and $U \rightarrow U^{\prime}$ we get

$$
\psi=c^{2} \frac{\partial G}{\partial v^{\prime}},
$$

where the right hand side is evaluated at $u^{\prime}=U, v^{\prime}=0$. As in an earlier example the actual value of the constant $c^{2}$ has no real significance. Since the Green's function for a quarter-plane is a source together with three image sources, the above form of $\psi$ can be interpreted as a doublet together with the image doublet in the line $u=0$. It is not surprising that the singularity in the hodograph plane should turn out to be of this type as this is indicated by the geometrical patterns of the streamlines. The solution for the limiting case as $U^{\prime} \rightarrow U$ can also be found by a Fourier sine transform where there is a delta function condition at the point $B$.

\section{The Extension to Compressible Flow}

The above examples serve to illustrate the use of direct hodograph methods in problems in two-dimensional incompressible flow. It is possible to quote many others. However, we wish now to turn to the problem of compressible flow and to show how a number of the above examples can be adapted immediately to solve analogous problems in compressible flow, provided the velocity in that flow is nowhere supersonic. This last condition is necessary to ensure that the differential equation remains elliptic so that the boundary value problems which occur (notably Dirichlet's problem) remain appropriate. This does not mean, of course, that extensions cannot be made to continue the flow into the supersonic region in certain cases but this is a problem of considerable difficulty and is outside the scope of our present discussion. We shall therefore limit ourselves to flow patterns in which only subsonic and sonic velocities appear.

It is well known that the equations of motion of a gas are non-linear in the physical plane but when $\psi$ is written as a function of the velocity variables the resulting equation is linear. The equation is

$$
4 \tau^{2}(1-\tau) \frac{\partial^{2} \psi}{\partial \tau^{2}}+4 \tau\{1+(\beta-1) \tau\} \frac{\partial \psi}{\partial \tau}+\{1-(2 \beta+1) \tau\} \frac{\partial^{2} \psi}{\partial \theta^{2}}=0
$$


where $\tau=q^{2} / q_{m}^{2}, q_{m}$ is the maximum velocity attainable by the gas, $\beta=1 /(\gamma-1)$ and $\gamma$ is the adiabatic index of the gas. As $\gamma \rightarrow \infty, \beta \rightarrow 0$ and Laplace's equation for incompressible flow is obtained.

The solution of (4) which is found by separating the variables is

where

$$
\psi_{n}(\tau) \sin (n \theta+\epsilon)
$$

$$
\psi_{n}(\tau)=\tau_{2}^{\frac{1}{2}{ }_{2}} F_{1}\left(a_{n}, b_{n} ; n+1 ; \tau\right),
$$

$a_{n}$ and $b_{n}$ being the roots of the equation

$$
2 t^{2}-2(n-\beta) t-\beta n(n+1)=0 .
$$

When $n$ is non-integral we get $\psi_{-n}(\tau) \sin (n \theta+\epsilon)$ as a "second solution". When $n$ is integral the second solution is complicated. However, many important problems which include all those we consider at present can be solved without recourse to the second solution. It is of importance to notice that $\psi=\theta$ is also a solution of (4) as it was a solution of Laplace's equation.

Suppose we consider the problems of figs. 1 and 2 with a gas instead of an incompressible fluid. The boundary conditions in the hodograph plane are exactly the same and the solution in the velocity variables is therefore

$$
\psi=\frac{4 K}{\pi} \sum_{k=0}^{\infty} \frac{1}{2 k+1} \frac{\psi_{4 k+2}(\tau)}{\psi_{4 k+2}\left(\tau_{0}\right)} \sin (4 k+2) \theta,
$$

where $\tau_{0}$ is the value of $\tau$ corresponding to $q=q_{0}$, the velocity of the jet far upstream. $K$ is slightly changed in value, being given by $K=h q_{0} \rho_{0} / \rho^{\prime}$, where $\rho_{0}$ is the density in the jet when $q=q_{0}$ and $\rho^{\prime}$ is the density at a stagnation point; as before $2 h$ is the width of the jet at a large distance upstream from the plate.

Similarly if we replace $(q / U)^{2 n}$ by $\psi_{2 n}(\tau) / \psi_{2 n}\left(\tau_{0}\right)$ in equation (2) we obtain the solution for the flow of a gas jet against a plate of finite width. We can also solve the Borda mouthpiece problem for gases and along with it a whole series of similar problems involving the efflux of a gas from a reservoir into a region of constant pressure. The principle is to replace $(q / U)^{k}$ where it occurs by $\psi_{k}(\tau) / \psi_{k}\left(\tau_{0}\right)$ and to leave $\theta$ unchanged. It should be emphasised, however, that this is not merely a tentative procedure the results of which are verified later, but the definite solution of a specific Dirichlet problem for equation (4). It happens that for these problems the solution can be obtained by superposing the solutions obtained by separating the variables. As the techniques of Green's functions and of transform methods for equation (4) are not so well developed as for Laplace's equation we are unable at present to solve the same range of problems as we are able to solve in incompressible flow. Problems such as that of figs. 4 and 5 do not have their analogues in compressible flow as the hodograph plane is always restricted to the region $q<q_{m}$. In practice, as we have already mentioned, we are restricted to the region $q \leqslant q^{*}$, where $q^{*}$ is the velocity corresponding to a Mach number of 1 , that is, to the region in which the equation is elliptic. However, it is of interest to make a fuller investigation of the properties of equation (4) with a view to developing for this equation some of the more advanced techniques by means of which the 
harder boundary value problems of Laplace's equation can be solved. The results of some work of this nature will shortly be the subject of another paper.*

Another important equation in the study of compressible flow is Tricomi's equation, which is an approximation of equation (4) valid for small perturbations about $q=q^{*}, \theta=0$. This is

$$
\frac{\partial^{2} \psi}{\partial \eta^{2}}+\eta \frac{\partial^{2} \psi}{\partial \theta^{2}}=0
$$

where $\eta$ is a certain dimensionless function of the velocity such that $\eta=0$ corresponds to the sonic velocity and $\eta=\infty$ is the approximation which represents the stagnation condition. Specifically, $\eta=(\gamma+1)^{\frac{1}{a}}\left(q^{*}-q\right) / q^{*}$ and $\eta \geqslant 0$ over the field. The solution which is found by separating the variables is

$$
\eta^{\frac{1}{2}} \mathbf{K}_{\frac{3}{3}}\left(\frac{2}{3} \lambda \eta^{\frac{\hat{z}}{2}}\right) \sin \lambda \theta
$$

where $K^{\frac{1}{3}}(z)$ is the modified Bessel function of order $\frac{1}{3}$.

It must be remembered that although mathematically we can solve problems of the type we have been considering in terms of these functions, the approximation has physical significance only for small values of $\theta$. Thus we cannot discuss this equation, for example, in connexion with the impact of a jet against a plane wall but we can consider a jet impinging on a wedge of small angle $\mu \pi$ and solve this problem. As far as mathematical techniques are concerned we are better equipped because the properties of Bessel functions and of Bessel's differential equation are well-known. The doublet type singularity corresponding to the flow at infinity has been correctly identified and a number of problems have been solved. The advantages arising from the appearance of Bessel functions in place of the much more complicated hypergeometric functions of the exact theory must be balanced against the limited range of applicability of the transomic approximation. For solutions similar to that given by equation (5) it is of interest to note that some algebraic simplification is achieved when the velocity at infinity is sonic as the term corresponding to $\psi_{n}\left(\tau_{0}\right)$ on the denominator is now

$$
\lim _{n \rightarrow 0} \eta^{\frac{1}{2}} K_{\frac{1}{3}}\left(\frac{2}{3} \lambda \eta^{\frac{3}{3}}\right)
$$

which is a constant multiple of $\lambda^{-t}$.

* Proc. Camb. Phil. Soc. (in the press).

Mathematics Department

St Salvator's College

UNIVERSITY OF ST ANDREWS 